bordés; stries très obsolètes, à part la suturale qui est surtout bien visible à la base. Trois pores sétigères sur la place du troisième inter-

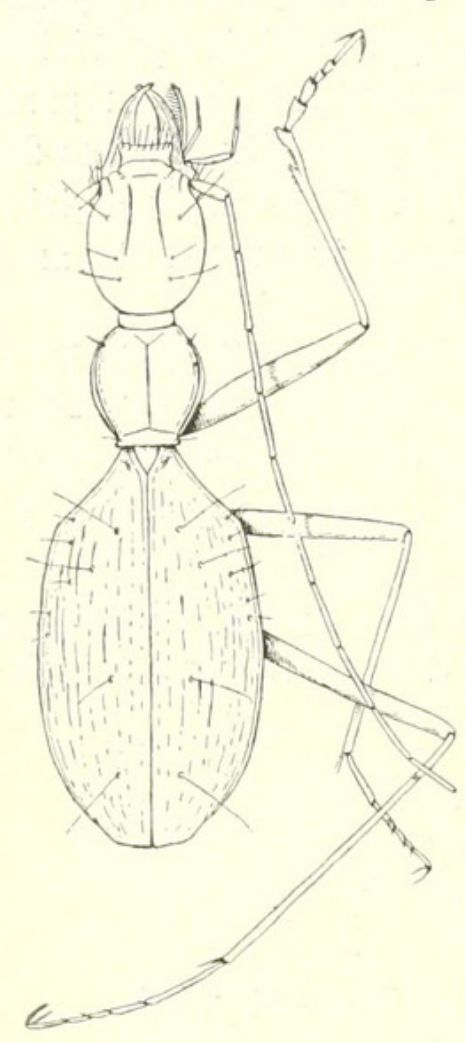

Fig. 1. - Aphaenops orionis Fagniez, $\times 8$.

valle, un autre sur la place du cinquième; une series umbilicata de cinq pores, dont le deuxième et le troisième sont reportés en dedans. Pattes allongées et pubescentes.

A. orionis est voisin d'A. Jeanneli A b., dont il se distingue par sa forme moins allongée, sa tête et surtout son protho-

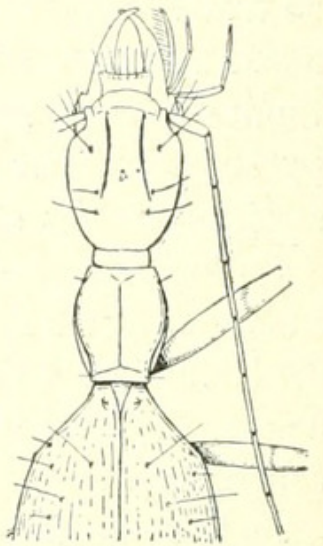

Fig. 2. - Aphaenops Jeanneli A b., $\times 8$. rax plus arrondis.

Pyrénées françaises: grotte de ChâteauPignon, à 14 kil. au sud de St-Jean-Piedde-Port, dans la commune de Saint-Michel (Basses-Pyrénées). Cette grotte s'ouvre à $1.030 \mathrm{~m}$. d'altitude, près de la forêt d'Orion (1).

Un individu pris le 12 août 1913, au cours d'une visite faite avec mon ami le $\mathrm{D}^{\mathrm{r}}$ JeAnNel, à qui je dois les deux dessins qui complètent si heureusement cette description.-

A. Jeanneli Ab. habite le bassin du gave de Mauléon, A. orionis celui de la Nive.

\title{
Sur deux Aphaenops nouveaux des Basses-Pyrénées
}

\section{[Col. Carabidae]}

par le $\mathrm{D}^{\mathrm{r}} \mathrm{R}$. JEANNel.

Aphaenops vasconicus, n. sp. - Long. 4,8 mill. - Voisin des A. Pandellei Lind. et A. Chaudoiri Bris., c'est-a-dire des espèces

(1) La description de cette grotte sera publiẻe prochainement dans la $5^{\mathrm{e}}$ série de Biospeologica. 
à corps pubescent et à prothorax très allongé. Forme convexe très etroite en avant; coloration brun testacé assez foncé; pubescence dorée longue, dense et redressée sur tout le corps. Les pores sétigères sont au nombre de $\mathbf{2}$ supraorbitaires, 2 sur les bords du pronotum, 3 discaux sur le troisième intervalle des élytres, deux près des bords en arrière de la ligne médiane; enfin la series umbilicata est désagrégée, très difficile à distinguer de la pubescence de l'élytre. Tête allongée, parallèle, avec un cou à peine distinct; le front après les antennes est deux fois aussi long que large. Les sillons frontaux sont longs, parallèles et se terminent dans le quart postérieur de la tête. Les mandibules sont extrêmement longues et grêles; le lobe interne de la maxille est presque aussi long que les mandibules. Antennes assez courtes, grêles, ne dépassant pas le milieu des élytres. Prothorax deux fois aussi long que large, à côtés arrondis et graduellement rétrécis jusqu'à la base, sans si-

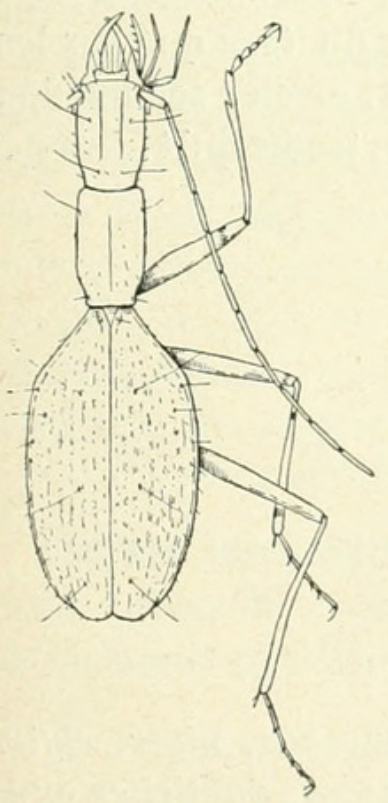

Fig. 2. - Aphaenops $\leftarrow$ ChaudoiriLind., $\times 8$. nuosité. Les côtés du

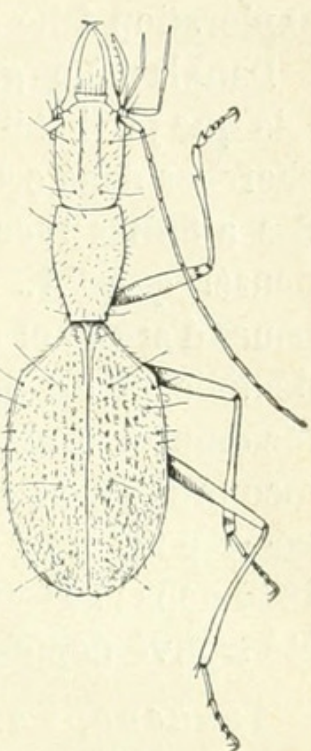

Fig. 1. - Aphaenops vasconicus Jeannel, $\times 8$. pronotum sont rebordés;

la ligne médiane est à peine indiquée, la base est très légèrement impressionnée; les angles sont mousses. Élytres ovales, avec les épaules saillantes, anguleuses; le bord huméral est tranchànt, très finement crénelé et cilié. La surface des élytres porte des stries obsolètes et très inégales, beaucoup plus rugueuses que chez A. Chaudoiri. Pattes courtes et grêles; les tibias postérieurs sont fortement arqués en dehors.

Cette nouvelle espèce rappelle $A$. Pandellei par la forme de son prothorax, A. Chaudoiri par son aspect général. Elle est facile à distinguer d'A. Pandellei par sa taille, sa pubescence, la forme de sa tête longue et parallèle au lieu d'être globuleuse, ses sillons frontaux parallèles tandis qu'ils sont divergents chez A. Pandellei; de plus, chez ce dernier les angles huméraux sont arrondis, nullement tranchants.

D'autre part, A. vasconicus est plus petit que l'A. Chaudoiri, sa 
pubescence est bien plus longue et fournie, la forme de son prothorax est toute différente et ses élytres sont beaucoup plus rugueux.

Basses-Pyrénées : grotte d'Oxibar, près de Camou-Cihigue; un exemplaire mâle pris sur une stalactite le 10 août 1913, au cours d'une exploration faite avec mon ami Ch. FAgniez.

Dans la même grotte se trouve l'A. Jeanneli Ab. C'était bien la dixième fois que je visitais la grotte d'Oxibar et c'est après huit heures de recherches dans cette petite grotte que j'ai trouvé ce nouvel Aphaenops. Ses affinités avec les $A$. Chaudoiri, A. Pandellei et $A$. Ehlersi me font penser que l'A. vasconicus est comme eux un habitant des grands bancs d'argile et que sa présence sur les stalactites d'Oxibar était accidentelle. Il est probable qu'il doit vivre dans des galeries argileuses inconnues, sous-jacentes à la grotte stalagmitée et qu'une cause inconnue l'avait attiré hors de son habitat normal. C'est vraisemblablement pour une raison analogue que l'A. Minos Lind. découvert en 1863 dans la grotte de Fontanet, près d'Ussat (Ariège) n'y a plus jamais été trouvé depuis.

Aphaenops orionis Fagniez(Bull. Soc.ent. Fr. [1913], p. 381). J'ai recueilli deux exemplaires de cette espèce nouvelle dans la grotte des sources de la Nive de Béhérobie, près de St-Jean-Pied-de-Port (Basses-Pyrénées). Ces exemplaires ne diffèrent du type que par leur taille qui est de $6, \breve{5}$ mill. (au lieu de 6,1 mill.). A. orionis semble donc bien être spécial aux grottes du bassin de la Nive, tandis qu'A. Jeanneli A b . peuple le bassin du gave de Mauléon.

\section{Description d'un Pelorurus Mars. [CoL. Histeridae] nouveau} par H. Desbordes.

Pelorurus Crampeli, n. sp. - Ovalis, convexus, niger, elytris viridi-coeruleis, tarsis brunneis. Frons parum concava, dupliciter punctulata, stria laterali. Pronotum lateribus fortiter punctatum, sed non ad basim, excepta punctorum linea ante scutellum; stria marginali lateribus et antice integra. Elytrorum striae profundae, subhumeralibus fere integris, 1-3 geminatis, 4-6 simplicibus, 4 integra, 5 dimidiata apicali, 6 integra; $2^{\circ}$ interstitio a medio ad basim valde dilatato. Pro- 


\section{$2 \mathrm{BHL}$ Biodiversity Heritage Library}

1914. "Sur deux Aphaenops nouveaux des Basses-Pyrenees." Bulletin de la Société entomologique de France 1913, 382-384.

https://doi.org/10.5962/bhl.part.16304.

View This Item Online: https://www.biodiversitylibrary.org/item/36377

DOI: https://doi.org/10.5962/bhl.part.16304

Permalink: https://www.biodiversitylibrary.org/partpdf/16304

\section{Holding Institution}

Smithsonian Libraries

\section{Sponsored by}

Smithsonian

\section{Copyright \& Reuse}

Copyright Status: NOT_IN_COPYRIGHT

This document was created from content at the Biodiversity Heritage Library, the world's largest open access digital library for biodiversity literature and archives. Visit BHL at https://www.biodiversitylibrary.org. 